\title{
Aprendizaje de conceptos químicos: una visión desde los trabajos prácticos y los estilos de aprendizaje
}

\section{Learning chemical concepts: a vision from some practical works and learning styles}

Rodrigo Rodríguez-Cepeda'

Recibido: abril 04 de 2016

Aceptado: junio 28 de 2016

\section{Resumen}

Es importante que los constructos conceptuales logrados durante la vida universitaria sean aplicados dentro del contexto de problemas sociales actuales. Sin embargo, son conocidas las dificultades de los profesionales recién egresados en el momento de aplicar los conceptos, demostrando inseguridad del conocimiento profesional, descontextualización frente a la realidad del entorno y desactualización de conceptos; esto a pesar de los esfuerzos por parte de profesores e investigadores, quienes han desarrollado nuevas formas de enseñanza alejadas de las tradicionales memorísticas. La presente investigación se centró en la construcción de conceptos químicos asociados a la cromatografía en el contexto de la química de alimentos, identificando las posibles relaciones entre los trabajos prácticos y los estilos de aprendizaje propuestos por Honey y Mumford. La muestra poblacional corresponde a los estudiantes de séptimo semestre de un programa de Licenciatura en Química y se emplea una metodología cuasiexperimental. Los resultados indican que la relación entre los trabajos prácticos y el aprendizaje de conceptos favorecen a los estudiantes con estilo de aprendizaje pragmático.

\begin{abstract}
It is very important that conceptual constructs achieved during college life can be applied within contexts of current social problems. However, difficulties of graduates for applying concepts are known, demonstrating insecurity of professional knowledge, isolation of environment reality, and out-of-date concepts. This happens, in spite of all efforts by professors and researchers at college, who have developed new ways of teaching, which are far distant from traditional memory-related approaches. In this context, this research focus on the construction of chemical concepts associated to chromatography within the context of food chemistry, identifying possible relations with the practical works and learning styles proposed by Honey and Mumford. The sample is seventhsemester students of a BA major in Chemistry, using a quasi-experimental design. The results indicate that the linking between the practical works and learning concepts benefits students with a pragmatic learning style.
\end{abstract}

Keywords: practical work, learning styles, chromatography, learning in context.

Palabras clave: trabajos prácticos, estilos de aprendizaje, cromatografía, aprendizaje en contexto. 


\section{Introducción}

La sociedad actual se caracteriza por su complejidad, diversidad y constantes cambios. Gracias a las nuevas tecnologías las personas se pueden comunicar con otras en cualquier parte del mundo, trayendo como consecuencia que la sociedad se esté aproximando a una cultura universal con problemáticas igualmente universales. Este fenómeno ha obligado a que las universidades sean los lugares donde los cambios se proyecten y se hagan visibles (Cruz, 2001); en este sentido, es necesario trabajar desde allí con el fin de desarrollar habilidades y actitudes en los estudiantes para poder afrontar los cambios en la sociedad.

Por otra parte, se perciben dificultades en muchos profesionales al emplear su conocimiento en la solución de los problemas que aquejan a la sociedad, no solo porque están inseguros de su conocimiento, sino porque están descontextualizados de la realidad cambiante de su entorno, razones por las cuales encuentran muchos obstáculos en el momento de proponer soluciones a dichas problemáticas. Esta circunstancia queda claramente expresada cuando el Business and Economics--Banking and Finance (2005), realiza una crítica a los profesionales colombianos mencionando que: "Los profesionales colombianos confían demasiado en su intuición, en sus habilidades para negociar, y en su malicia indígena, mientras que los profesionales de otras latitudes hacen bien la tarea - estudiando las opciones, evaluando con rigor las propuestas y preparando exhaustivamente los cursos de acción" (Financieras, 2005).

De acuerdo con lo anterior, es necesario que en la universidad los estudiantes desarrollen las habilidades necesarias para tomar decisiones importantes en su futuro profesional basados en un conocimiento contextualizado; sin embargo, esto será posible si el profesor asume una postura activa frente a sus estudiantes (González, 2001), especialmente durante la intervención en el aula, de tal forma que aproxime el conocimiento a las realidades sociales de los estudiantes, sus expectativas frente al aprendizaje y la forma como aprenden. El profesor debe diseñar estrategias didácticas encaminadas a lograr un aprendizaje para proponer soluciones a los problemas sociales del futuro profesional (Sánchez-Blanco \& ValcárcelPérez, 2000).

En el contexto anterior, el presente artículo muestra los resultados de una intervención de aula, con estudiantes de séptimo semestre del programa de Licenciatura en Química de la Universidad Pedagógica Nacional, registrados en el curso de Métodos de Análisis Químico II. La finalidad del curso es identificar las posibles relaciones entre los trabajos prácticos y los estilos de aprendizaje en la construcción de conceptos básicos asociados a la cromatografía, como son: fase estacionaria, fase móvil, tiempos de retención y clases de cromatografía, entre otros, en el contexto de la química de alimentos.

\section{Marco teórico y metodología}

\subsection{Referente conceptual}

i) Trabajos Prácticos

Los trabajos prácticos en el laboratorio de química han sido reconocidos como una estrategia que permite desarrollar en los estudiantes algunas habilidades del saber hacer en contexto. Según Rodríguez-Zambrano (2007), los trabajos prácticos involucran algunos aspectos como:

I. Capacidad de internalizar conocimientos (saber- conocer).

II. Conocimientos generales y específicos (saberes).

III. Destrezas técnicas y procedimentales (saber-hacer).

IV. Desarrollo de actitudes (saber-ser).

V. Competencias sociales (saber-convivir). 
Por otra parte, se acepta que los trabajos prácticos generan motivación en los estudiantes, permiten un conocimiento vivencial de fenómenos, correlacionan variables influyentes en el fenómeno y permiten acercarse a las metodologías y procedimientos propios de la investigación de los científicos, generan un ambiente propicio para el trabajo en equipo y el desarrollo de actitudes como la planificación, orden, limpieza y seguridad en el laboratorio, entre otras (Jiménez, Caamaño, Oñorbe, \& Pedrinaci, 2007).

Además, los trabajos prácticos tienen ciertas dificultades, especialmente cuando son planeados como ejercicios cerrados, los cuales pierden efectividad, y el estudiante solamente sigue una serie de pasos sin un objetivo definido (AngaritaVelandia, Fernández-Morales, \& Duarte, 2011); por ello se han propuesto diferentes formas de elaborar trabajos prácticos de carácter abierto, donde la observación libre de los estudiantes les permite enfrentarse a situaciones nuevas que deben resolver y por tanto, les permite construir nuevos conceptos (Catalá, Cubero, Díaz, Feu, \& Jiménez, 2007).

Atendiendo la necesidad antes planteada, Jiménez et al. (2007) proponen clasificar los trabajos prácticos según su objetivo en:

I. Experiencias: Destinados a obtener una familiarización perceptiva con los fenómenos.

II. Experimentos Ilustrativos: Destinados a ilustrar un principio o relación entre variables. Suponen una aproximación cualitativa o semicuantitativa del fenómeno.

III. Ejercicios prácticos: Diseñados para aprender determinados procedimientos para realizar experimentos que ilustren o corroboren la teoría.

IV. Investigaciones: Diseñadas para promover en los estudiantes las metodologías puestas en acción por los científicos en la resolución de problemas, las cuales permiten el desarrollo de habilidades, destrezas y procedimientos propios de la indagación.

De la misma manera, los trabajos prácticos pueden ser planteados según el contexto, de tal forma que los estudiantes comprendan la relación entre el conocimiento científico y la actividad humana; así, logran un acercamiento a las diferentes miradas sobre la aplicación de la ciencia y pueden desarrollar actitudes críticas frente a ellos (Cañal et al., 2011).

\section{ii) Estilos de Aprendizaje}

Definir estilos de aprendizaje puede no ser fácil debido a que este concepto no tiene un consenso entre autores y es definido de forma muy variada en las investigaciones. La mayoría coinciden en afirmar que el estilo es "la forma como la mente procesa la información o como es influida por las percepciones de cada individuo" (Baldwin, Reckers, \& Kolb, 1984 ; Kolb, 1976; Mativo, Hill, \& Godfrey, 2013; Williams, Brown, \& Etherington, 2012). Algunos autores describen el estilo de aprendizaje como "algunas capacidades de aprender que se destacan por encima de otras como resultado del aparato hereditario, de las experiencias vitales propias y de las exigencias del medio ambiente actual" (Capella \& Coloma, 2003).

Debido a esta variedad de definiciones, es posible encontrar diferentes propuestas para definir estilos de aprendizaje, entre los cuales se destacan varios investigadores relacionados en la tabla 1 : 


\begin{tabular}{|c|c|}
\hline Autor & Estilo de aprendizaje \\
\hline Felder \& Silverman (1988) & $\begin{array}{cl}\text { Información percibida: } \\
-\quad \text { Sensitivos } \\
-\quad \text { Intuitivos } \\
\text { Percepción sensitiva: } \\
-\quad \text { Visuales } \\
-\quad \text { Verbales } \\
\text { Organización de la información: } \\
-\quad \text { Inductivos } \\
-\quad \text { Deductivos } \\
\text { Comprensión de la información: } \\
-\quad \text { Secuenciales } \\
-\quad \text { Globales } \\
\text { Forma de trabajar la información: } \\
-\quad \text { Activos } \\
-\quad \text { Reflexivos }\end{array}$ \\
\hline Fleming \& Mills (1992) & $\begin{array}{l}\text { Visual } \\
\text { Auditivo } \\
\text { Lector/Escritor } \\
\text { Quinestésico }\end{array}$ \\
\hline Honey \& Mumford (1986) & $\begin{array}{l}\text { Reflexivo } \\
\text { Teórico } \\
\text { Pragmático } \\
\text { Activo }\end{array}$ \\
\hline Grasha \& Reichmann (1974) & $\begin{array}{l}\text { Competitivo } \\
\text { Colaborativo } \\
\text { Participativo } \\
\text { Dependiente } \\
\text { Independiente } \\
\text { Evitante }\end{array}$ \\
\hline
\end{tabular}

Tabla 1. Clasificación de estilos de aprendizaje.

Los autores mencionados en la tabla 1 han realizado sus investigaciones centradas en los individuos y proponen un aprendizaje individual y propio de cada persona, lo cual implica que los estudiantes llevan al aula una complicada configuración de rasgos personales, orientaciones específicas hacia la vida y al mundo, igualmente, cuentan con un conjunto particular de destrezas y actitudes educativas (González, 2001).

De esta manera, el modelo de los estilos de aprendizaje propuesto por Honey \& Mumford (1986), citados por Capella \& Coloma, (2003), facilita un diagnóstico de los alumnos con un nivel más técnico y objetivo que la simple observación asistemática (Domingo \& Antonio, 2008); dicha propuesta ofrece datos acerca de cómo prefieren aprender los alumnos, si necesitan más o menos dirección y estructura. Lo anterior permite a los docentes contar con una interesante información para tomar decisiones acerca de la selección de materiales educativos, cómo presentar la información, creación de grupos y subgrupos de trabajo, procedimientos de evaluación, etc.

El presente trabajo se fundamenta en la clasificación de los estilos de aprendizaje dados por Honey \& Mumford (1986), donde las principales características de cada uno de ellos se describen en la Tabla 2. 


\begin{tabular}{lllll}
\hline Características & \multicolumn{1}{c}{ Activo } & \multicolumn{1}{c}{ Reflexivo } & \multicolumn{1}{c}{ Teórico } & \multicolumn{1}{c}{ Pragmático } \\
\hline Principales & Animador & Ponderado & Metódico & Experimentador \\
& Improvisador & Concienzudo & Lógico & Práctico \\
& Descubridor & Receptivo & Objetivo & Directo \\
& Arriesgado & Analítico & Crítico & Eficaz \\
& Espontáneo & Exhaustivo & Estructurado & Realista \\
Otras & Creativo & Observador & Disciplinado & Técnico \\
& Novedoso & Recopilador & Planificado & Útil \\
& Renovador & Paciente & Sistemático & Rápido \\
& Inventor & Cuidadoso & Ordenado & Decidido \\
& Innovador & Detallista & Sintético & Planificador \\
& Conversador & Estudioso & Razonador & Concreto \\
& Líder & Investigador & Pensador & Objetivo \\
& Participativo & Asimilador & Perfeccionista & Claro \\
& Competitivo & Prudente & Generalizador & Organizador \\
\hline
\end{tabular}

Tabla 2. Características de los estilos de aprendizaje según Honey y Mumford. Tomado y adaptado de (Capella y Coloma, 2003).

El estudio de los estilos de aprendizaje permite conocer mejor a los estudiantes, de tal manera que las actividades desarrolladas en el aula se pueden diseñar basadas en dichas particularidades (Lozano, 2006); esta metodología toma mayor importancia cuando los estudiantes presentan dificultades en la construcción de los conceptos. La presente investigación pretende aportar en la identificación de relaciones entre los estilos de aprendizaje descritos por Honey y Mumford, y los trabajos prácticos de laboratorio a través del aprendizaje de conceptos asociados a la cromatografía en el contexto del análisis de alimentos.

\subsection{Metodología}

El diseño metodológico de la investigación se fundamenta en los principios cuasi experimentales sin grupo control. La muestra poblacional objeto de estudio se conformó por 25 estudiantes de Licenciatura en Química de la Universidad Pedagógica Nacional, registrados en el séptimo semestre, correspondiente al ciclo de profundización. Para identificar los estilos de aprendizaje se aplicó el cuestionario CHAEA diseñado por Alonso (1992), basado en el modelo de Honey y Mumford (1986); se indagan los preconceptos en cromatografía; posteriormente, se diseñan y aplican actividades que incluyen trabajos prácticos de laboratorio organizados secuencialmente.

Inicialmente los estudiantes realizan consultas en fuentes bibliográficas recomendadas por el profesor acerca de algunos conceptos básicos de cromatografía, especialmente sobre los fenómenos físicos de adsorción, reparto, intercambio iónico y exclusión molecular. Esta actividad previa pretende lograr una reflexión conceptual, mediante la indagación, el lenguaje escrito, la libre expresión y el respeto por las ideas de los demás.

Posteriormente se forman grupos a discreción de los estudiantes, se debate alrededor de los fenómenos fisicoquímicos que intervienen en la cromatografía; cada grupo define los tópicos según sus intereses, escriben sus ideas en un papel y luego las comparte con los compañeros, quienes complementan las ideas. Después se procede a la socialización en la que cada grupo expone sus temáticas con las respectivas explicaciones, correcciones y aclaraciones de dudas mediante una interacción entre estudiantes y profesor.

Luego del debate se efectúan los trabajos prácticos, los cuales se plantean en el contexto 
de la química de alimentos, de tal forma que se proponen protocolos para determinar vitamina C mediante cromatografía HPLC y la presencia de colorantes artificiales empleando cromatografía en capa fina; esto permite crear un espacio para plantear problemas, hipótesis, trabajos prácticos de laboratorio y explicaciones por parte del profesor.

Para finalizar, se complementa la evaluación del proceso de construcción conceptual por parte de los estudiantes mediante la aplicación de un instrumento final, el cual indaga sobre conceptos asociados a la cromatografía.

\section{Resultados y discusión}

La identificación de los estilos de aprendizaje es una herramienta útil para adaptar la metodología de enseñanza del docente, a la vez que permite diseñar métodos de evaluación apropiados para determinar el avance de los estudiantes (Acevedo, Tirado, \& Montero, 2015); en este sentido, los resultados obtenidos en la aplicación del cuestionario CHAEA para identificar los estilos de aprendizaje se muestran en la figura 1.

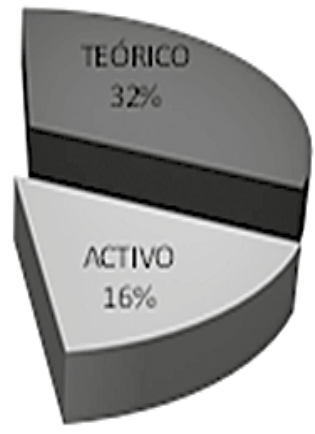

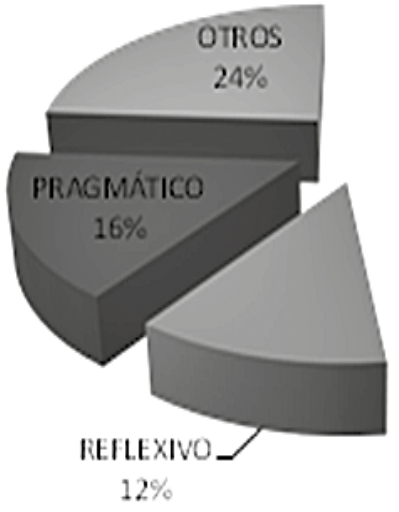

Figura 1. Resultado de la aplicación del cuestionario CHAEA: estilos de aprendizaje en estudiantes de Licenciatura en Química.

De acuerdo con la figura 1 es posible concluir que los estudiantes del ciclo de profundización de la carrera de Licenciatura en Química, registrados en séptimo semestre, presentan diversidad de estilos de aprendizaje, con una mayor tendencia al estilo teórico con el $32 \%$ de la población. Se presume que estos estudiantes adaptan e integran las observaciones en teorías lógicas, su pensamiento es secuencial, proceden paso a paso e integran hechos a teorías coherentes, analizan y sintetizan la información, no les gusta los juicios subjetivos, buscan la racionalidad y objetividad (Alonso, Gallego, \& Honey, 1997; Capella \& Coloma, 2003).
Es importante resaltar la dificultad de categorizar al $24 \%$ de los estudiantes en algún estilo de aprendizaje, debido a la multiplicidad de características propias de los diversos estilos de aprendizaje. Por ello se propone incluir una nueva categoría denominada multiestilo, en la cual se ubicaría este grupo de estudiantes, quienes seguramente se adaptan a cualquier actividad que se les proponga, hipótesis que debe ser estudiada a profundidad en otra investigación.

De acuerdo con lo anterior, es posible suponer que la mayoría de estudiantes son metódicos, críticos, estructurados, sistemáticos, pensadores, perfeccionistas, entre otras cualidades, que están 
enumeradas en la tabla 2; igualmente, se presume que las actividades a diseñar deben presentar situaciones estructuradas, con una finalidad clara, tener la posibilidad de cuestionar, leer o escuchar sobre ideas que insisten en la racionalidad (Alonso, Gallego, \& Honey, 1997).

\section{i) Instrumento inicial, indagación conceptual}

Una vez identificados los estilos de aprendizaje, se aplica una prueba inicial, previamente validada, diseñada por Chaparro, Pérez, \& Ramírez (2011), la cual consta de ocho preguntas e indaga sobre conceptos básicos de cromatografía y se resume en la tabla 3.

\section{Intencionalidad de pregunta}

\section{Objetivo}

1. Indaga sobre las técnicas de separación de Pretende identificar si los estudiantes reconocen el mezclas. método de separación.

2. Indaga sobre las fases de la cromatografía.

Pretende verificar la identificación de las fases móvil y estacionaria.

3. Indaga sobre los diferentes tipos de Reconoce los diferentes tipos de cromatografía. cromatografía.

4. Indaga sobre las aplicaciones de las diferentes técnicas cromatográfícas.

Pretende observar el lenguaje utilizado para justificar la respuesta.

5. Indaga sobre los fenómenos físicos que suceden en una cromatografía.

Pretende observar el lenguaje utilizado para justificar la respuesta.

6. Identificación de un esquema de cromatografía. Reconoce las partes que integra un cromatógrafo de gases.

7. Indaga en la aplicación de la cromatografía de gases.

Determinar el grado de reconocimiento de la cromatografía de gases.

8. Indaga sobre la cromatografía HPLC.

Determinar el nivel de conocimiento de la cromatografía HPLC.

Tabla 3. Resumen de la prueba inicial.

En la Figura 2 se observa el resultado de la aplicación del instrumento resumido en la tabla 2. 


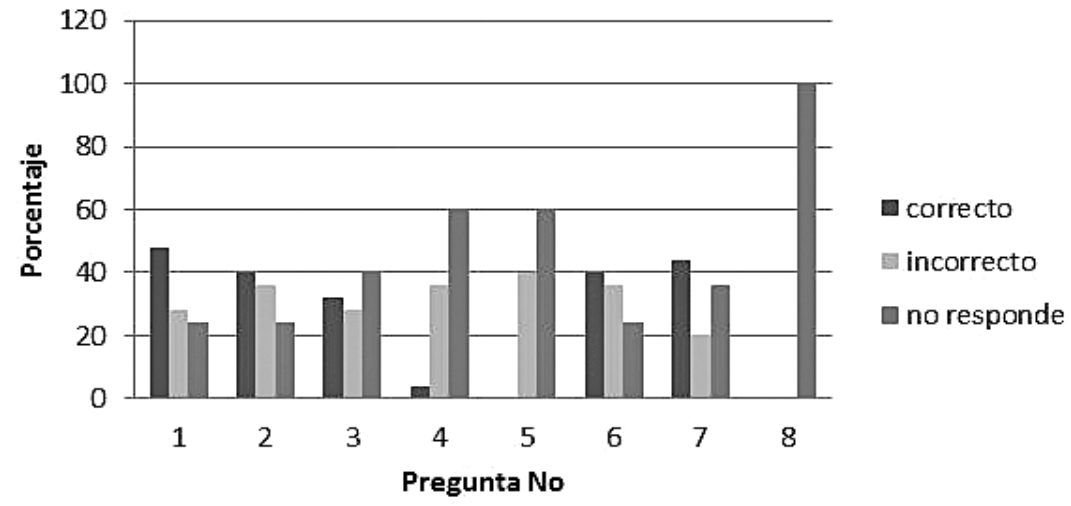

Figura 2. Resultado de la aplicación pre prueba conceptual.

De acuerdo con la figura 2 , se destacan los resultados de las preguntas 1 a 3,6 y 7 , las cuales muestran que entre un 31 y un 46 por ciento de los estudiantes logran responder correctamente sobre conceptos básicos relacionados con cromatografía. Sin embargo, existe un alto porcentaje de estudiantes que responden incorrectamente o no responden las preguntas, llegando a valores hasta del $100 \%$ al avanzar en el cuestionario, como se observa en la figura 2 para la pregunta 8.

Los resultados obtenidos refuerzan la necesidad de identificar herramientas didácticas que mejoren los procesos de aprendizaje de conceptos adecuados, donde los trabajos prácticos surgen como una alternativa viable para tal fin, especialmente si son diseñados teniendo en cuenta los estilos de aprendizaje.

\section{ii) Aplicación de la estrategia}

En una primera etapa se realiza una clase magistral por parte del profesor, a fin de presentar los conceptos de cromatografía, fenómenos físicos involucrados y su explicación matemática, usos y clases de cromatografía; se incluye un ejercicio de preguntas a los estudiantes sobre algunos de estos conceptos, encontrándose algunas respuestas erróneas, como se muestra en la tabla 4.

\begin{tabular}{ll}
\hline \multicolumn{1}{c}{ Concepto } & \multicolumn{1}{c}{ Ejemplo de Respuesta } \\
\hline Adsorción & $\begin{array}{l}\text { Es un fenómeno físico que se observa cuando un líquido se } \\
\text { incorpora dentro de otra sustancia. }\end{array}$ \\
Intercambio iónico & $\begin{array}{l}\text { Cuando al formarse un compuesto se intercambian } \\
\text { electrones entre los átomos. } \\
\text { Reparto }\end{array}$ \\
\hline
\end{tabular}

Tabla 4. Descripción de conceptos antes del debate. 
Una vez contextualizados, se forman grupos de estudiantes quienes trabajan los conceptos presentados en la tabla 4, mediante consultas en libros y artículos; se desarrolla un debate en torno a dichas temáticas y se elaboran conclusiones.

Con el debate se logran algunas claridades conceptuales con altos niveles de certeza en las argumentaciones dadas respecto a cada concepto abordado; desde este punto de vista, puede reconocerse la habilidad de buscar teorías, elaborar cuestionamientos y discursos coherentes, lo cual coincide con lo expuesto por Capella y Coloma,(2003).
Por otro lado, se observan altos niveles de receptividad frente a las aclaraciones de las dudas a pesar de las complicaciones surgidas para algunos de los estudiantes en la comprensión y aplicación de los conceptos a tratar; el debate permite aclarar inquietudes mediante un trabajo conjunto de asesoría del profesor y opiniones de los compañeros. Algunas de las conclusiones logradas en este ejercicio frente a algunos conceptos asociados a la cromatografía, se resumen en la tabla 5:

\begin{tabular}{ll}
\hline \multicolumn{1}{c}{ Concepto } & \multicolumn{1}{c}{ Ejemplo de Respuesta } \\
\hline Adsorción & La adsorción es cuando un líquido es retenido sobre la \\
& superficie del material sólido. \\
& El intercambio iónico es cuando los componentes de la \\
Intercambio lónico & muestra se distribuyen entre las fases, según su carga \\
& iónica, siendo adsorbidos o diluidos por la fase móvil o \\
& estacionaria según su afinidad. \\
Reparto & En el reparto los componentes de las mezclas se reparten \\
& entre la fase móvil y la fase estacionaria por diferencia de \\
& solubilidades. \\
\hline
\end{tabular}

Tabla 5. Descripción de conceptos durante el debate.

Si bien a las respuestas dadas por los estudiantes aún les falta profundidad, es claro el avance conceptual al comparar las respuestas de la tabla 4 con las de la tabla 5; esto es gracias a la manera como está estructurada la actividad, la cual permite mejorar la forma de describir los conceptos asociados a cromatografía. Por lo anterior, la construcción colectiva de conceptos mediante acuerdos parece ser una vía eficaz, reforzando lo mencionado por Capella y Coloma (2003), quienes plantean la riqueza que existe en estos ejercicios al motivar el cambio de personas pensadoras a personas con habilidades de crear relaciones.

iii) Trabajos Prácticos
Una vez alcanzadas las claridades conceptuales durante el debate, se aplican los trabajos prácticos cuya finalidad es plantear situaciones problema que permitan reafirmar los conceptos teóricos construidos utilizando como contexto el análisis de alimentos.

En el primer trabajo práctico se planea un análisis por cromatografía líquida HPLC, donde se pide definir la metodología analítica para determinar vitamina $C$ en un alimento seleccionado por cada grupo; de esta manera, los estudiantes deben indagar para proponer las condiciones cromatográficas, se incentivan a aplicar los conocimientos adquiridos durante el debate, como son: reparto, adsorción, y fase reversa entre otros. 
Posteriormente, con asesoría del profesor se realizan los ajustes pertinentes a la metodología analítica y se trabaja en el laboratorio; este ejercicio permite identificar algunas dificultades de los estudiantes al proponer metodologías analíticas, así como para plantear problemas, hipótesis y definir objetivos; igualmente, en un $65 \%$ de los casos, se observan problemas con los cálculos matemáticos para la preparación de reactivos, confunden las unidades de concentración y los factores de dilución, conceptos que deben ser abordados durante el desarrollo del trabajo práctico.

Con el desarrollo de los protocolos en el laboratorio, cada grupo analiza el contenido de vitamina $C$ (ácido ascórbico) en una fruta cítrica mediante cromatografía HPLC, para lo cual describen los fenómenos químicos y físicos de cada etapa del protocolo, desde la extracción hasta la obtención del cromatograma y los cálculos relacionados como concentración, uso de factores de dilución, exactitud y precisión, entre otros.
Los estudiantes plantean interrogantes como: ¿Qué fuerzas están presentes en el proceso de separación?; ¿Cómo son los procesos de retenciónadsorción en la columna cromatográfica?; ¿Cuál es la razón por la que existen tantos tipos de cromatografía y si son complementarios o se contraponen?; ¿Cuál es la diferencia de colocar un líquido o un gas en un equipo de cromatografía de gases o en uno HPLC?; ¿De qué depende que se muestren unos picos más rápida o más lentamente?

Estos cuestionamientos y las respuestas dadas, ver figura 3, muestran que los trabajos prácticos permiten profundizar en los conceptos asociados a la cromatografía gracias a la motivación de los estudiantes por indagar, proponer preguntas y dar respuesta a las mismas (González-Aguado, MiyarAlacano, Gil-Martín, Mentxaka-Bearan, \& SantosCañas, 2002), igualmente, es clara la interiorización de la ciencia y cómo se hace ciencia al emplear los trabajos prácticos (Rodríguez-Rey, Real-García, \& Gregori-Labarta, 2015).

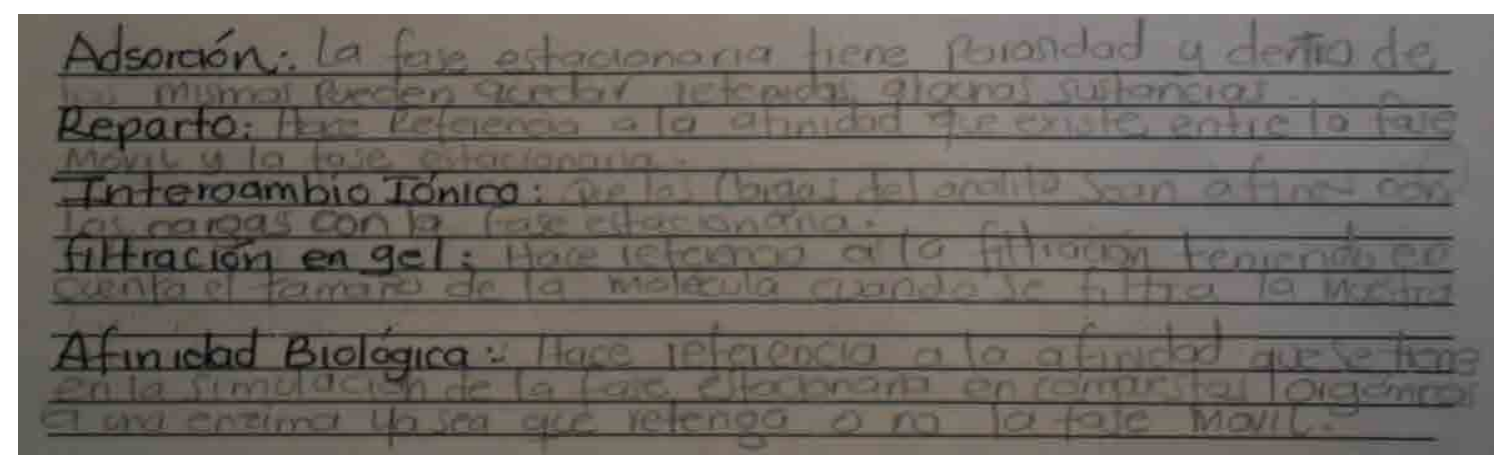

Figura 3. Argumentación frente a fenómenos que se dan en cromatografía.

iv) Instrumento Final

Como cierre de la intervención se aplica un instrumento final similar al inicial y con los mismos objetivos planteados por cada pregunta, ver tabla 3 , el cual permite determinar el avance conceptual de los estudiantes; en la figura 5 se muestran los resultados de la aplicación. 


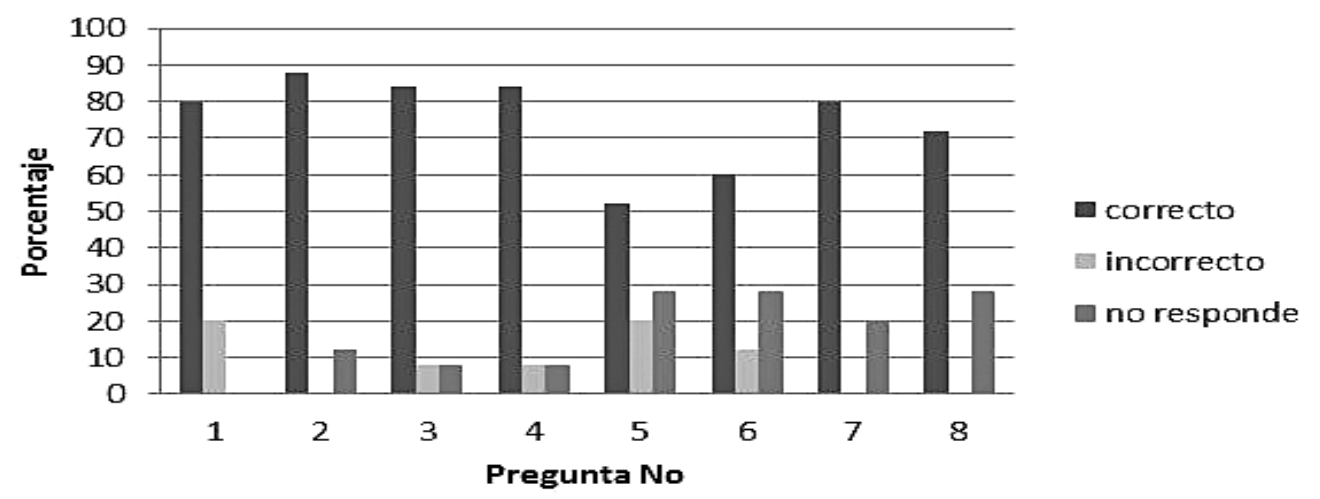

Figura 5. Resultado de la aplicación pos prueba conceptual.

La prueba permite realizar una evaluación en dos vías, siendo la primera el determinar el nivel de acierto en la respuesta dada a cada pregunta. La figura 5 muestra que los porcentajes de acierto son elevados, destacándose la pregunta 2 con un $88 \%$; esta pregunta pretende identificar el nivel de entendimiento sobre las características diferenciadoras entre las técnicas cromatográficas; también se destaca la pregunta 5 con un 52\% de aciertos, la cual identifica el entendimiento de los fenómenos físicos que se presentan en la cromatografía.
Adicionalmente, se genera una evaluación cuantitativa en una escala entre 0 a 5 , donde cinco es el valor más alto, con esta valoración se definen tres categorías así: Bajo desempeño 0 a 3; Desempeño medio 3 a 4 y Alto desempeño 4 a 5. En la figura 6 se observa que el $35 \%$ de los estudiantes con alto desempeño pertenecen al estilo pragmático, mientras que no hay estudiantes con estilo reflexivo.

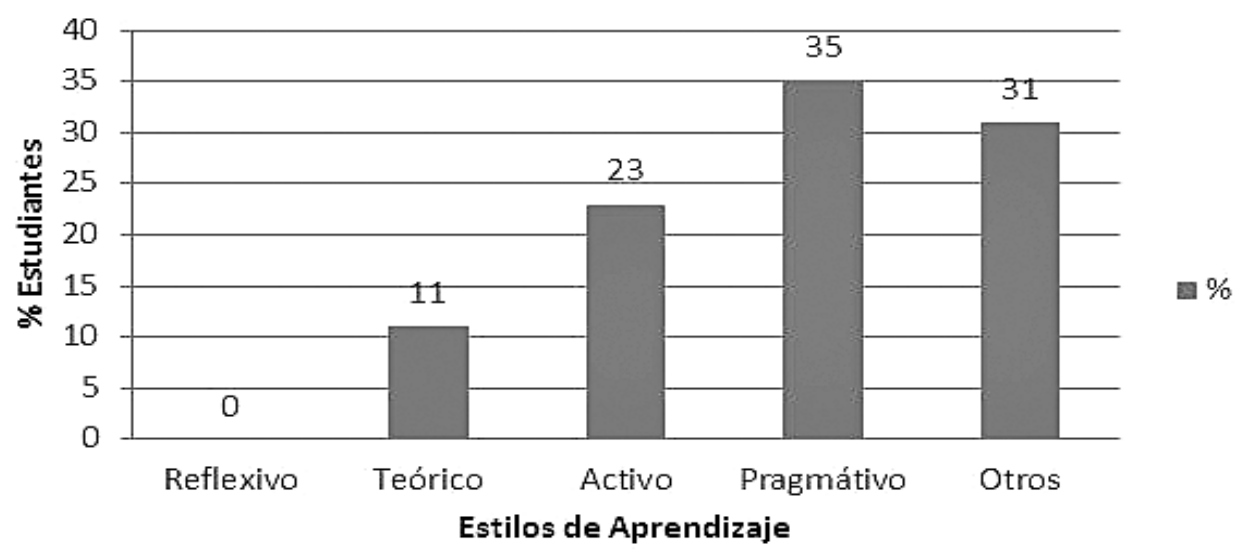

Figura 6. Porcentaje de estudiantes con desempeño alto y su estilo de aprendizaje.

De acuerdo con los resultados, se puede mencionar el efecto positivo generado por las actividades realizadas, las cuales favorecen la construcción de conceptos asociados a la cromatografía, con mayor incidencia en los estudiantes con estilo de aprendizaje pragmático. 
Lo anterior permite reforzar lo planteado por algunos investigadores frente a la necesidad de fomentar en los estudiantes las habilidades de proponer hipótesis y probar experimentalmente soluciones a problemas, indagando las características propias de cada individuo a fin de actuar en consecuencia (Cárdenas \& Montealegre, 2001). Los resultados también muestran la imperiosa necesidad de desarrollar metodologías de enseñanza pertinentes, las cuales deben tener en cuenta las habilidades, preferencias y estilos de aprendizaje de los estudiantes, factores relevantes en el proceso de aprendizaje (Guanipa \& Mogollón, 2006).

\section{Conclusiones}

La aplicación de las actividades propuestas develan dificultades conceptuales por parte de los estudiantes de Licenciatura en Química de la Universidad Pedagógica Nacional, registrados en el séptimo semestre, especialmente frente a conceptos básicos de cromatografía, como: reparto, adsorción, fase móvil e intercambio iónico, entre otros. Lo anterior justifica la pertinencia de diseñar estrategias didácticas encaminadas a favorecer la construcción de conceptos, teniendo en cuenta las particularidades de cada estudiante; para ello es útil la aplicación del cuestionario CHAEA, que permite categorizar la muestra poblacional desde la clasificación de los estilos de aprendizaje, propuestos por Honey y Mumford (1986).

Se encontró al estilo de aprendizaje teórico como el de mayor predominancia y el reflexivo como el menos predominante. Se destaca un porcentaje importante de estudiantes, 24\%, que tienen características de todos los estilos de aprendizaje, por lo que se propone clasificarlos en una nueva categoría denominada multiestilo.

Frente a las actividades planteadas, la indagación y el debate se destacan como herramientas importantes para contextualizar a los estudiantes en el tema de cromatografía, especialmente por el intercambio de opiniones y aclaraciones entre estudiantes y profesor.

Por su parte, los trabajos prácticos favorecen la construcción de conceptos relacionados con la cromatografía debido a su efecto motivador; además, se permite a los estudiantes plantear hipótesis, generar preguntas y teorías con el fin de contrastarlas y probarlas en el laboratorio.

Los resultados finales muestran que los estudiantes con estilo pragmático logran los mejores rendimientos, probablemente por la afinidad entre las actividades planteadas y las características individuales enmarcadas en el estilo de aprendizaje. En este sentido, se muestra que planear la enseñanza teniendo en cuenta las particularidades de cada estudiante, permite aumentar las posibilidades de éxito en el aprendizaje de conceptos asociados a la cromatografía.

\section{Agradecimientos}

El autor agradece al Doctorado Interinstitucional en Educación de las Universidades Pedagógica Nacional, Universidad Distrital Francisco José de Caldas y Universidad del Valle, por la posibilidad de desarrollar la presente investigación en el marco de su Tesis Doctoral.

\section{Referencias}

Acevedo, D., Tirado, D., \& Montero , P. (2015). Perfil de aprendizaje y rendimiento académico en una asignatura de química en modalidad a distancia y presencial en dos programas de ingeniería. Formación Universitaria, 8 (6), 39-46. doi: 10.4067/ S0718-50062015000600006

Alonso, C. (1992). Estilos de aprendizaje: análisis y diagnóstico en estudiantes universitarios. Madrid, España: Universidad Complutense. 
Alonso, C., Gallego, D., \& Honey, P. (1997). Los estilos de aprendizaje. Procedimientos de diagnóstico y mejora. Madrid, España: Ediciones mensajero.

Angarita-Velandia, M.A., Fernández-Morales, F.H., \& Duarte, J.E. (2011). Utilización de material didáctico para la enseñanza de los conceptos de ciencia y tecnología en niños. Revista de Investigación, Desarrollo e Innovación, 2 (1), 35-43. Recuperado de: http://revistas.uptc.edu.co/revistas/index.php/ investigacion_duitama/article/view/1307

Baldwin, B., Reckers, P., \& Kolb, D. (1984). Exploring the role of learning style research in accounting education policy. Journal of Accounting Education, 2 (2), 63-76. doi: 10.1016/0748-5751(84)90006-X

Cañal, P., García, S., Jiménez, M., Márquez, C., Martínez, C., \& Pedrinaci, E. (2011). Didáctica de la biología y la geología. Barcelona, España: Graó.

Capella, R., \& Coloma, M. (2003). Estilos de aprendizaje. Lima, Perú: Fondo Editorial Pontificia Universidad Católica.

Cárdenas, F., \& Montealegre, R. (2001). Miniprojects: an alternative to improve general chemistry teaching in higher education. Journal of Science Education, 2 (2), 100 - 02. Recuperado de: http:// search.proquest.com/openview/0501d5ccc3e288 02c0414ac37b214695/1?pq-origsite=gscholar

Catalá, M., Cubero, R., Díaz, J., Feu, M., \& Jiménez, P. (2007). Las ciencias en la escuela: teorías y prácticas. Barcelona, España: Graó.

Chaparro, P., Pérez, P., \& Ramírez, A. (2011). Diseño e implementación de una unidad didáctica para el aprendizaje de conceptos asociados a la cromatografía: una visión desde los estilos de aprendizaje. Bogotá, Colombia: Universidad Pedagógica Nacional.
Cruz, D. (2001). Enseñanza aprendizaje en la educación superior: un reto para el siglo XXI. Humacao, Puerto Rico: Universidad de Puerto Rico.

Domingo , G., \& Antonio, L. (2008). Los estilos de aprendizaje y la enseñanza de las matemáticas. Revista Complutense de Educación, 19 (1), 95-112. Recuperado de: http://revistas.ucm.es/index.php/ RCED/article/viewFile/RCED0808120095A/15564

Felder, R., \& Silverman, L. (1988). Learning and teaching styles in engineering education. Engineering Education, 78 (7), 674-681. Recuperado de: http://s3.amazonaws.com/ academia.edu.documents/31039406/LS-1988. pdf?AWSAccessKeyld=AKIAJ56TQJRTWSMT NPEA \&Expires $=1478184978 \&$ Signature $=$ NO s8al4bOV0cwLvyiJSfApTTUmw\%3D\&respo nse-content-disposition $=$ inline $\% 3 B \% 20$ filename\%3DLearning_and_teaching_styles_in_ engineer.pdf

Financieras, N. (02 de febrero de 2005). OpiniónDebilidades colombianas frente a la competencia global. El Tiempo. Recuperado de: http://www. eltiempo.com/archivo/documento/MAM1631821

Fleming, N., \& Mills, C. (1992). Not another inventory, rather a catalyst for reflection. To Improve the Academy, 11, 137-143. Recuperado de: http:// digitalcommons.unl.edu/cgi/viewcontent.cg i? article $=1245 \&$ context $=$ podimproveacad $\& s$ ei-redir $=1$ \& referer $=$ http $\% 3 \mathrm{~A} \% 2 \mathrm{~F} \% 2 \mathrm{Fscholar}$. google.com. co\%2Fscholar\%3Fq\%3DNot\%2 Banother\%2Binventory\%2C\% 2Brather\%2Ba\% 2Bcatalyst\%2Bfor\% 2Breflection $\% 26 \mathrm{hl} \% 3$ Des\%26as_sdt\%3D0\%26as_vis\%3D1\%26oi\%3 Dscholart\%26sa\%3 DX\%26ved\%3D0ahUKEwj4ff54zQAhVHsVQKHVDjBp8QgQMIGTAA \#search= \%22Not\%20another\% 20inventory\% 2C\%20 rather\% 20catalyst\%20reflection $\% 22$

González, F. (2001). Los estilos de aprendizaje de los estudiantes y los estilos de enseñanza de los 
profesores: hacia un modelo de concienciación. Río Piedras, Puerto Rico: Universidad de Puerto Rico.

González-Aguado, M.E., Miyar-Alacano, M.C., GilMartín, A., Mentxaka-Bearan, I., \& Santos-Cañas, M.T. (2002). Una experiencia de implantación de los medios informáticos en los laboratorios de ciencias. Journal of Science Education, 3 (2), 92-94. Recuperado de: http://search.proquest.com/ope nview/26c4025143d126eb868e3e0500349edd/1? pq-origsite $=$ gscholar $\& \mathrm{cbl}=28899$

Grasha, A., \& Reichmann, S. (1974). A rational approach to developing and assessing the construct validity of a student learning style scales instrument. The Journal of Psycology: Interdisciplinary and Applied, 87 (2), 213-223. doi: 10.1080/00223980.1974.9915693

Guanipa, M., \& Mogollón, E. (2006). Estilos de aprendizaje y estrategias cognitivas en estudiantes de ingeniería. Revista Ciencias de la Educación, 1 (27), 11-27. Recuperado de: http://servicio.bc.uc. edu.ve/educacion/revista/volln27/27-1.pdf

Honey, P., \& Mumford, A. (1986). The manual of learning styles. Maindehead, Reino Unido: Peter Honey.

Jiménez, M., Caamaño, A., Oñorbe, A., \& Pedrinaci, E. (2007). Los trabajos prácticos en ciencias. Barcelona, España: Graó.

Kolb, D. (1976). Management and the learning process. California Management Review, 18 (3), 2131.
Lozano, A. (2006). Estilos de aprendizaje y enseñanza: un panorama de la estilística educativa. México, D.F., México: Trillas.

Mativo, J., Hill, R., \& Godfrey, P. (2013). Effects of human factors in engineering and design for theaching mathematics: a comparison study of online face-to-face at tecnical college. Journal of STEM Education, 14 (4), 3644. Recuperado de: http://www.jstem.org/ index.php?journal $=\quad$ JSTEM \&page $=$ article \&op $=$ view\&path $\% 5 \mathrm{~B} \% 5 \mathrm{D}=1830$

Rodríguez-Rey, C., Real-García, J.J., \& GregoriLabarta, I. (2015). El aprendizaje de la metodología científica a través de trabajos prácticos. Revista Didáctica, Innovación y Multimedia, 11 (32), 1-20. Recuperado de: https://dialnet.unirioja.es/servlet/ articulo?codigo $=5321847$

Rodríguez-Zambrano, A. (2007). El paradigma de las competencias, hacia la educación superior. Revista de la Facultad de Ciencias Económicas , XV (1), 151. Recuperado de: http://www.redalyc.org/ articulo.oa?id=90915108

Sánchez-Blanco, G., \& Valcárcel-Pérez, M. V. (2000). ¿Qué tienen en cuenta los profesores cuando se selecciona el contenido de enseñanza?, cambios y dificultades tras un programa de formación. Enseñanza de las Ciencias, 18 (3), 423-437. Recuperado de: http://ddd.uab.cat/record/1503

Williams, B., Brown, T., \& Etherington, J. (2012). Learning styles of undergraduate nutrition and dietetics students. Journal Allied Heald, 41 (4), 170-176. Recuperado de: http:// www.ingentaconnect.com/content/asahp/ jah/2012/00000041/00000004/art00006 